\title{
Clinical and radiological long-term outcome after posterior cruciate ligament reconstruction and nonanatomical popliteus bypass
}

\author{
Tom Adler • Niklaus F. Friederich • Felix Amsler • \\ Werner Müller • Michael T. Hirschmann
}

Received: 22 June 2014 / Accepted: 21 August 2014 / Published online: 17 September 2014

(C) SICOT aisbl 2014

\begin{abstract}
Purpose The purpose of this study was to analyse the longterm outcome of patients treated for combined posterior cruciate ligament $(\mathrm{PCL})$ and posterolateral corner injuries by combined PCL reconstruction and popliteus bypass according to Mueller or refixation of the popliteus tendon.

Methods Sixteen patients treated by combined PCL reconstruction and popliteus bypass according to Mueller $(n=7)$ or refixation of the popliteus tendon $(n=9)$ were included. A mean follow-up of $24 \pm$ three years was performed using the International Knee Documentation Committee (IKDC) 2000, Lysholm, Tegner and the Knee Injury and Osteoarthritis Outcome (KOOS) scores. Bilateral stress radiographs were performed. The degree of osteoarthritis was assessed using Kellgren Lawrence score. Pearson correlations of predictive factors for worse outcome were performed $(p<0.05)$.

Results Categorically, total IKDC 2000 was B (nearly normal) in five (31\%), C (abnormal) in seven (44\%) and D (severely abnormal) in four ( $25 \%$ ) patients. Lysholm score was $68 \pm 22$; KOOS symptom score was $40 \pm 13$, KOOS pain $26 \pm 24$, KOOS activity $18 \pm 18$, KOOS sport $51 \pm 32$ and KOOS LQ 44 \pm 26 . Median Tegner score decreased from pre-injury 7 (range 4-10) to 4 (range 2-10) at follow-up.
\end{abstract}

T. Adler $\cdot$ N. F. Friederich $•$ W. Müller $\cdot$ M. T. Hirschmann $(\bowtie)$

Department of Orthopaedic Surgery and Traumatology,

Kantonsspital Baselland-Bruderholz, CH-4101 Bruderholz,

Switzerland

e-mail: michael.hirschmann@ksbl.ch

M. T. Hirschmann

e-mail: Michael Hirschmann@web.de

M. T. Hirschmann

e-mail: Michael.Hirschmann@unibas.ch

F. Amsler

Amsler Consulting, Basel, Switzerland
Kellgren Lawrence score showed minimal osteoarthritis in seven (44\%), moderate osteoarthritis in seven (44\%) and severe osteoarthritis in one $(12 \%)$ patient.

Conclusions The challenging group of patients treated by PCL reconstruction and popliteus bypass according to Mueller et al. or popliteus refixation showed only moderate clinical and radiological long-term outcome without statistical difference, even if patient age at surgery and the long-term follow-up is acknowledged. Anatomical posterolateral corner reconstruction techniques should be preferred.

Keywords Posterior cruciate ligament reconstruction . Popliteus bypass $\cdot$ Popliteus tendon refixation $\cdot$ Outcome

\section{Introduction}

Posterior cruciate ligament (PCL) injuries are frequently combined with posterolateral corner (PLC) injuries [1-3]. The incidence of acute PCL injuries varies between $3.4 \%$ and $20 \%$ [4], and the incidence of isolated PLC injuries is less frequent $[2,5-7]$. Despite improved diagnostic possibilities, these injuries are still often missed [8-11]. When missed, these injuries in particular are known to lead to development of early osteoarthritis (OA), most likely in the medial tibiofemoral and patellofemoral joints [12]. This finding has been explained by constant overloading of the patellofemoral and medial tibiofemoral joints (reversed Maquet effect) [4, 13]. It is also known that missed lesions of peripheral structures, such as the PLC, can cause instability and then lead to a subsequent early failure of PCL reconstruction $[1,14,15]$. This being emphasised, a variety of repair and reconstruction techniques, both nonanatomical and anatomical, have been reported over recent decades [14, 16-21]. Nonanatomical techniques, such as Clancy, Hughston/Jacobson and Mueller $[14,18,21]$ were used initially. Increasingly, anatomical 
reconstruction techniques, such as Chang, Larson, LaPrade and Miyatake $[16,17,19,20]$ are used.

One nonanatomical technique used in the early days of reconstructive knee surgery is the popliteus bypass according to Mueller. In this technique, a 1-cm-wide strip of the iliotibial tract is harvested, shuttled through a tibial tunnel and attached on the former femoral attachment of the popliteus tendon (Fig. 1) [21]. To date, there are no reported studies investigating the long-term outcome after combined PCL reconstruction and popliteal bypass or popliteal tendon repair.

The primary purpose of our study was to analyse the longterm outcome of patients treated for combined PCL and PLC injuries using combined PCL reconstruction and popliteus bypass according to Mueller or combined PCL reconstruction and refixation of the popliteus tendon.

\section{Methods}

A review of the medical records in in the Bruderholz Hospital archives was performed. All patients who underwent a PCL reconstruction and an additional reconstruction of the PLC (popliteus bypass according to Mueller or refixation) between 1981 and 1992 were followed up for a minimum of 18 years. During this period, 47 patients were treated; 22 could not be located, one died and seven refused to participate. One patient with a knee reconstruction sustained a periprosthetic femoral fracture before the follow-up and refused to be included. Ultimately, 16 patients (male:female $=11: 5$, mean age $32 \pm 14$ years, weight $80 \pm 16 \mathrm{~kg}$, and height $175 \pm 9 \mathrm{~cm}$ at surgery) were included. All patients underwent an open PCL reconstruction using ipsilateral quadriceps tendon autograft. Of those 16 patients, nine (mean age at surgery $41 \pm 14$ years, mean follow-up $21 \pm$ two years) were treated with a popliteus tendon refixation and seven (mean age at surgery $22 \pm$ three years, mean follow-up $27 \pm$ two years) with the popliteus bypass.

Fig. 1 Popliteus bypass according to Mueller
Follow-up was carried out $23 \pm$ three [mean \pm standard deviation (SD)] years after surgery, including bilateral, standardised, weight-bearing radiographs [anteroposterior (AP), lateral, Rosenberg views [22]). In addition, bilateral anterior and posterior stress radiographs (in $30^{\circ}$ and $90^{\circ}$ flexion [2]) and varus stress radiographs were performed. Analysis of the AP stress radiographs was done according to Stäubli [23]. The extent of knee OA was graded according to the Kellgren-Lawrence OA score (0 normal; grade 1 suspected; grade 2 minimal; grade 3 moderate, grade 4 severe). All measurements were made using the Picture Archiving and Communications System (PACS) (Phillips Easy Vision, The Netherlands). Informed consent was obtained from all patients, and the study was approved by the local ethics committee (EK 295/09).

For clinical outcome assessment, we used the International Knee Documentation Committee (IKDC 2000) Standard Evaluation Form, the Lysholm score, the Tegner score and the Knee Injury and Osteoarthritis Outcome Score (KOOS). Clinical examination was carried out according to the IKDC 2000 form. In addition, anterior and posterior KT-1000 arthrometer measurements (Medmetric, San Diego, CA, USA) were performed with the knee in $25^{\circ}$ of flexion.

Statistical analysis was performed by a professional statistician using SPSS 17.0 (SPSS, Chicago, IL, USA). Continuous variables were described using mean, SD and range. Categorical variables were tabulated as absolute and relative frequencies. For statistical analysis, two groups were investigated (group 1, bypass; group 2, refixation). There were no statistical differences between patients in both groups. Traffic accidents were the main reason for injury in both groups (ten of 16; $62.5 \%$ ), followed by injuries while doing sports activities (five of 16, $31.3 \%$ ). A two-tailed Pearson's correlation was used to compute associations between variables. For all analyses, $p<0.05$ was considered statistically significant and $p<0.1$ as a tendency.

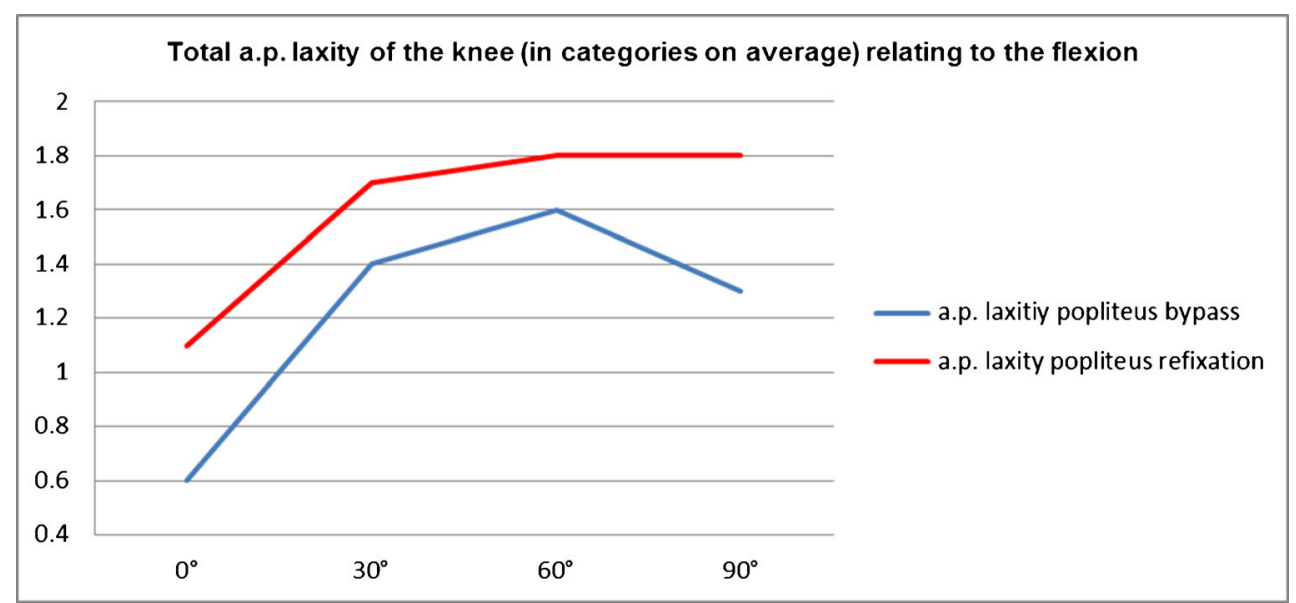




\section{Results}

Total IKDC 2000, IKDC 2000 for subcategories ligament and function, and Lysholm, Tegner and KOOS scores are presented in Table 1.

The side-to-side differences of anterior KT-1000 arthrometer measurements $134 \mathrm{~N}$ were $5.2 \pm 4 \mathrm{~mm}$ and of posterior KT-1000 arthrometer measurements were $1.6 \pm$ $1.5 \mathrm{~mm}$. A trend to a higher difference in anterior and posterior translation was recognised in group 1 compared with group 2 , although these differences were not significant due to the small number of patients. The Kellgren-Lawrence score showed minimal OA in seven (44\%), moderate OA in seven (44\%) and severe OA in one (12\%) patient. One patient had undergone a total knee replacement. In comparison with the contralateral side, the injured knee joint had a worse OA grade on average. To each $45 \%$ a suspicious or minimal OA was seen. Moderate OA was found in $9 \%$ only. According to the reverse Maquet effect [4, 13], the medial knee joint and patellofemoral joints showed worse OA than lateral knee joints.

The side-to-side difference of posterior stress radiographs at $30^{\circ}$ was $4 \pm 5 \mathrm{~mm}$ and $6 \pm 3 \mathrm{~mm}$ at $90^{\circ}$ flexion. Breakdown into two groups showed a trend $(p>0.05)$ toward increased posterior instability at $30^{\circ}(4.5 \pm 4.1$ vs. $3.7 \pm 6.4)$ and $90^{\circ}(8 \pm$ 2.4 vs. $3.7 \pm 3.1$ ) for group 2 . In contrast, side-to-side difference in bilateral varus stress radiographs was $0.4 \pm 2.3 \mathrm{~mm}$ in group 1 and $-0.5 \pm 2.6 \mathrm{~mm}$ in group 2. A similar trend $(p>0.05)$ was indicated for the Dial test, with a side-to-side difference of $3^{\circ} \pm 4.5^{\circ}$ at $30^{\circ}$ flexion and $4.1^{\circ} \pm 7^{\circ}$ at $90^{\circ}$ for group 1 and $0^{\circ} \pm 0^{\circ}$ plus $1.1^{\circ} \pm 3.1^{\circ}$, respectively, in group 2 .
Knees in group 1 showed less laxity on average while testing manually than the knees in group 2 . In addition, a decrease in laxity at $90^{\circ}$ of flexion in group 1 was recognised (Fig. 2).

\section{Discussion}

To our knowledge, there is no other clinical long-term study investigating patients after combined PCL reconstruction and popliteus bypass or popliteus tendon refixation due to combined PCL and PLC injury. The most important findings of this study are twofold: Firstly, clinical outcome measurements showed only moderate to poor results, and groups did not differ significantly. In addition, a more severe OA and a higher degree of laxity were detected in comparison with other studies [24-27]. However, the average follow-up in this study was 24 years and, thus, clearly longer than in the other studies.

When compared with more modern anatomical reconstruction techniques, our patients showed inferior clinical and radiological results. However, there is no single study with such a long follow-up. Kim et al. [28] evaluated patients treated with single-bundle PCL reconstruction combined with a PLC reconstruction according to LaPrade. Patients were divided into two groups: one with a conventional PCL reconstruction and the other with a left remnant of the PCL in addition. Their follow-up was, on average, 49 and 45 months, respectively. Moreover, their patients were significantly younger [39 (range 14-62) years] than patients in our study. As expected, those authors reported better results than for patients in our study. However, Tegner score showed a worse result at

Table 1 Subjective and objective outcome scoring of patients at final follow-up

\begin{tabular}{llll}
\hline Outcome instrument & all: mean $\pm \mathrm{SD}($ median, range) & group 1: mean \pm SD (median, range) & group 2: mean \pm SD (median, range) \\
\hline IKDC subjective & $40 \pm 22(31,12-84)$ & $35 \pm 23(30,14-68)$ & $39 \pm 22(41,12-84)$ \\
Total IKDC & $\mathrm{n}(\%)$ & $0(0 \%)$ & $0(0 \%)$ \\
A (normal) & $0(0 \%)$ & $2(29 \%)$ & $3(33 \%)$ \\
B (nearly normal) & $5(31 \%)$ & $3(43 \%)$ & $4(44 \%)$ \\
C (abnormal) & $7(44 \%)$ & $2(29 \%)$ & $2(22 \%)$ \\
D (severely abnormal) & $4(25 \%)$ & $69 \pm 22(74,27-93)$ & $67 \pm 23(77,33-90)$ \\
Lysholm & $68 \pm 22(76,27-93)$ & $-(7,4-10)$ & $-(7,4-10)$ \\
Tegner preinjury & $-(7,4-10)$ & $-(4,3-6)$ & $-(4,2-10)$ \\
Tegner at follow-up & $-(4,2-10)$ & $40 \pm 8(39,25-50)$ & $40 \pm 17(36,14-68)$ \\
WOMAC symptoms & $40 \pm 13(39,14-68)$ & $23 \pm 24(17,0-67)$ & $28 \pm 25(25,0-69)$ \\
WOMAC pain & $26 \pm 24(21,0-69)$ & $13 \pm 15(4,0-35)$ & $22 \pm 20(19,0-54)$ \\
WOMAC activities & $18 \pm 18(15,0-54)$ & $21 \pm 15(14,7-42)$ & $28 \pm 19(20,8-61)$ \\
WOMAC total & $25 \pm 17(20,7-61)$ & $46 \pm 30(30,15-85)$ & $55 \pm 34(65,0-100)$ \\
KOOS sports/leisure & $51 \pm 31(58,0-100)$ & $43 \pm 24(56,6-69)$ & $44 \pm 28(38,13-94)$ \\
KOOS quality of life & $44 \pm 26(44,6-94)$ & $26 \pm 16(24,9-49)$ & $32 \pm 21(25,7-66)$ \\
WOMAC/KOOS total & $30 \pm 19(24,7-66)$ & & \\
\hline
\end{tabular}

WOMAC Western Ontario and McMaster Universities Osteoarthritis Index, KOOS Knee Injury and Osteoarthritis Outcome Score, SD standard deviation 
Fig. 2 Total anteroposterior (AP) laxity of manually tested knees. Category 0, 0-2 mm; category 1 , $3-5 \mathrm{~mm}$; category $2,6-10 \mathrm{~mm}$; category $3,>10 \mathrm{~mm}$
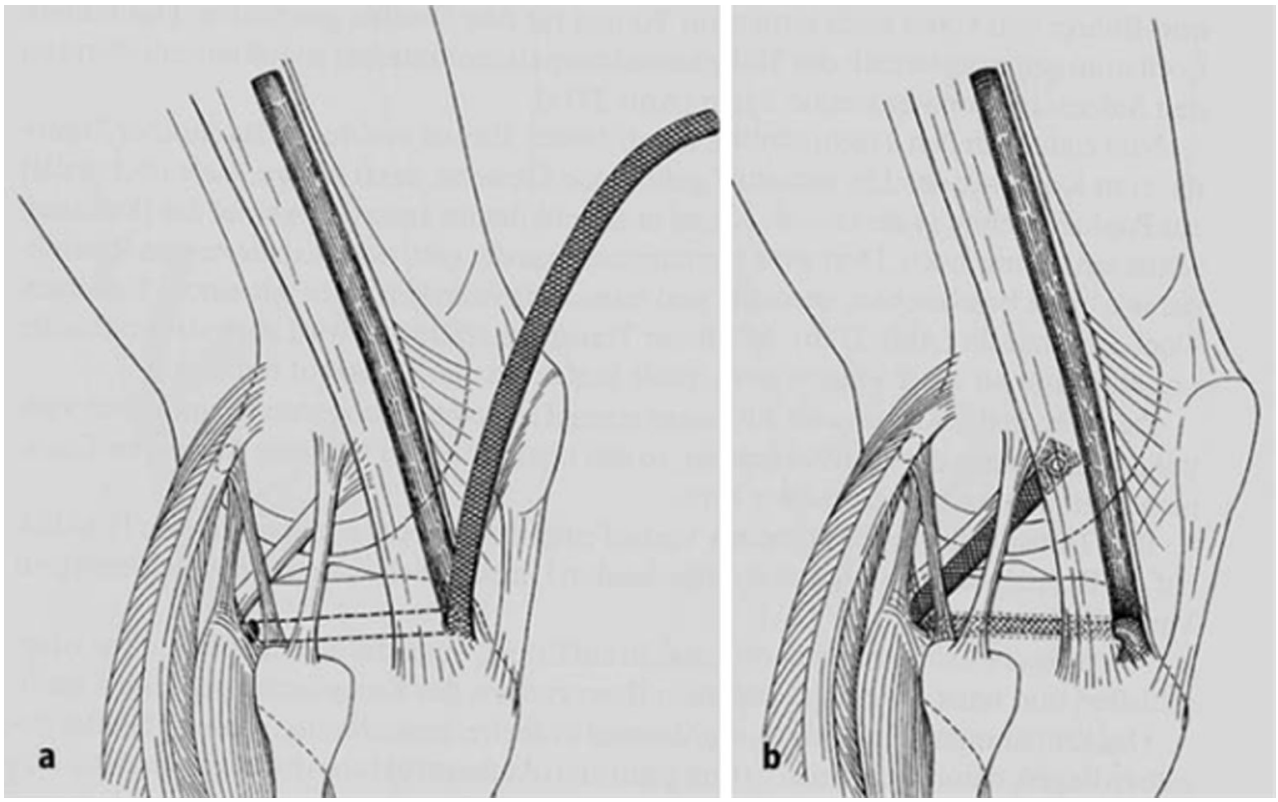

the last follow-up, which is most likely due to the inferior Tegner score before the injury.

Yoon et al. [29] reported a study with 17 patients (mean age 37 years) with a mean follow-up of 40 months after posterolateral reconstruction according to LaPrade plus additional reconstruction of the PCL or anterior cruciate ligament (ACL). Average Lysholm score was 85 in comparison with the Lysholm score of in our study, with 68 on average. Moreover, their median Tegner score was higher -6 at final follow-up-versus 4.2 in our study.

Thirty-nine months after a posterolateral reconstruction using a modified Larson technique $(n=24$, mean age 19 years at time of surgery), Rios et al. [30] indicated better results in Lysholm (83 on average) and Tegner (6 on average) scores. However, at least one cruciate ligament was reconstructed additionally. Freeman et al. [31] published a series of 17 patients (mean age 31 ; range $21-47$ years) on average 35 months after PCL or combined PCL/PLC reconstruction using the Larson technique. With an average Lysholm score of 69 and a Tegner score of 4.5 on average, these patients had similar results to our patients. However, the authors of that study stated that the PCL reconstruction alone showed worse results.

Interestingly, all the previous studies have a remarkably shorter follow-up and younger patients at the last follow-up compared with our study. Most of those studies showed superior results to ours, but Freeman et al. [31] showed comparable results in the Tegner and Lysholm scores. In summary, the inferior results found in our study are mainly due to a significantly longer average follow-up of 24 years. Another reason might be that a considerable number of patients complained of chronic instabilities.
The popliteus bypass according to Mueller might provide better stability than refixing the popliteus tendon, which might be explained by augmented static stability. This is in contrast to Fanelli [32], who stated that the popliteus bypass only gives stability in knee extension based on the missing dynamic component of the popliteus muscle. However, the popliteus bypass is considered an anisometric and nonanatomical reconstruction technique, a fact that could be the reason for inferior results of our study when compared with studies investigating outcomes after anatomical PLC reconstructions. Stannard [33] found in 56 patients with a 24-month follow-up that anatomical reconstruction showed better results than PLC refixation. In summary, PLC reconstruction techniques provide better outcome than refixation techniques.

Anatomical techniques might be superior than the presented nonanatomical, popliteus bypass technique. However, regardless of the differences between techniques, the main reason for inferior results of the study presented here is the significantly longer follow-up than all other mentioned studies. Secondly, the medial tibiofemoral as well as the patellofemoral knee compartment showed a higher degree of OA than the lateral tibiofemoral knee compartments. This finding is clearly in accordance with Lobenhoffer et al. [12], who showed that patients with posterior instability are more likely to develop OA of the medial tibiofemoral and patellofemoral knee joint ten to 20 years after PCL injury. Our study results confirm their findings. Furthermore, unaffected contralateral knee joints in our patients presented a less severe OA than the operated knee joints.

This study has several limitations: The small number of patients is the biggest limitation, but this is because evaluating long-term outcomes is difficult. Due to the small sample size, 
statistical comparison of both groups must be considered with due care.

\section{Conclusion}

The challenging group of patients with combined PCL and PLC injuries treated by PCL reconstruction and popliteus bypass according to Mueller et al. or popliteus refixation showed only moderate clinical and radiological outcome, even when patient age at surgery and long-term follow-up is acknowledged. In summary, long-term results of treated combined PCL/PLC injuries are not satisfying. The type of PLC reconstruction using these two methods did not show any statistically significant difference in outcomes. A trend was recognised that the popliteus bypass leads to less laxity than does refixation, although a statistically significant difference could not be found. However, in order to restore PLC instabilities, anatomical techniques should be preferred over nonanatomical techniques, such as popliteus bypass according to Mueller presented in this study.

\section{References}

1. Harner CD, Vogrin TM, Hoher J, Ma CB, Woo SL (2000) Biomechanical analysis of a posterior cruciate ligament reconstruction. Deficiency of the posterolateral structures as a cause of graft failure. Am J Sports Med 28:32-39

2. Malone AA, Dowd GS, Saifuddin A (2006) Injuries of the posterior cruciate ligament and posterolateral corner of the knee. Injury 37(6): 485-501

3. Vinson EN, Major NM, Helms CA (2008) The posterolateral corner of the knee. AJR Am J Roentgenol 190(2):449-458

4. Hoffman F (2004) Posterior knee instability. Orthopade 33(1):87104

5. Covey DC (2001) Injuries of the posterolateral corner of the knee. J Bone Joint Surg Am 83-A(1):106-118

6. Duri ZA, Aichroth PM, Zorrilla P (1997) The posterior cruciate ligament: a review. Am J Knee Surg 10(3):149-164

7. Ricchetti ET, Sennett BJ, Huffman GR (2008) Acute and chronic management of posterolateral corner injuries of the knee. Orthopedics 31(5):479-488

8. Hirschmann MT, El Rabadi J, Mueller C, Friederich NF (2009) Fixierte hintere Schublade nach VKB-Ersatzplastik bei scheinbar isolierter vorderer VKB-Ruptur. Orthopade 38(7):632-637

9. Hochstein P, Schmickal T, Grützner PA, Wentzensen A (1999) Diagnostic and incidence of the rupture of the posterior cruciate ligament. Unfallchirurg 102(10):753-762

10. Strobel MJ, Weiler A (2006) Therapie der chronischen HKB-Läsion. Arthroskopie 19:243-257

11. Strobel MJ, Weiler A, Schulz MS, Russe K, Eichhorn HJ (2002) Fixed Posterior Subluxation in Posterior Cruciate Ligament-Deficient Knees. Am J Sports Med 30:32-38

12. Lobenhoffer P (1999) Chronic instability after posterior cruciate ligament injury. Tactics, techniques, and results. Unfallchirurg 102(11):824-838
13. Skyhar M, Warren RF, Ortiz G, Schwartz E, Otis J (1993) The effect of sectioning of the posterior cruciate ligament and the posterolateral complex on the articular contact pressures within the knee. J Bone Joint Surg 75-A:694-699

14. Bach BR, Jewell BF, Dworsky B (1993) Posterolateral knee reconstruction using Clancy bizeps tenodesis. Surgical technique. Am J Knee Surg 6(3):97-103

15. LaPrade RF, Muench C, Wentorf F, Lewis JL (2002) The effect of injury to the posterolateral structures of the knee on force in a posterior cruciate ligament graft: A biomechanical study. Am J Sports Med 30:233-238

16. Chang CB, Seong SB, Lee S, Yoo JH, Park YK, Lee MC (2007) Novel methods for diagnosis and treatment of posterolateral rotatory instability of the knee. J Bone Joint Surg Am 89(Suppl 3):2-14

17. Fanelli GC, Larson RV (2002) Practical management of posterolateral instability of the knee. Arthroscopy 18(2 Suppl 1): $1-8$

18. Hughston JC, Jacobson KE (1985) Chronic posterolateral rotatory instability of the knee. J Bone Joint Surg Am 67(3):351-359

19. LaPrade RF, Johansen S, Wentorf FA, Engebretsen L, Ester-berg JL, Tso A (2004) An analysis of an anatomical posterolateral knee reconstruction: An in vitro biomechanical study and development of a surgical technique. Am J Sports Med 32:1405-1414

20. Miyatake S, Kondo E, Tsai TY, Hirschmann M, Halewood C, Jakobsen BW, Yasuda K, Amis AA (2011) Biomechanical comparisons between 4-strand and modified Larson 2-strand procedures for reconstruction of the posterolateral corner of the knee. Am J Sports Med 39(7):1462-1469

21. Müller W (1982) Das Knie. Form, Funktion und ligamentäre Wiederherstellungschirurgie. Springer, Berlin Heidelberg New York

22. Lobenhoffer P, Lattermann C, Krettek C, Blauth M, Tscherne H (1996) Rupture of the posterior cruciate ligament: status of current treatment. Unfallchirurg 99(6):382-399

23. Stäubli HU, Jakob RP (1990) Posterior instability of the knee near extension. A clinical and stress radiographic analysis of acute injuries of the posterior cruciate ligament. J Bone Joint Surg (Br) 72:225-230

24. Cooper DE, Stewart D (2004) Posterior cruciate ligament reconstruction using single-bundle patella tendon graft with tibial inlay fixation: 2- to 10-year follow-up. Am J Sports Med 2:346-360

25. Gerhard P, Bolt R, Dück K, Mayer R, Friederich NF, Hirschmann MT (2013) Long-term results of arthroscopically assisted anatomical single-bundle anterior cruciate ligament reconstruction using patellar tendon autograft: are there any predictors for the development of osteoarthritis? Knee Surg Sports Traumatol Arthrosc 21(4):957-964

26. Hirschmann MT, Iranpour F, Müller W, Friederich NF (2010) Surgical treatment of complex bicruciate knee ligament injuries in elite athletes: what long-term outcome can we expect? Am J Sports Med 38(6):1103-1109

27. Hirschmann MT, Zimmermann N, Rychen T, Candrian C, Hudetz D, Lorez LG, Amsler F, Müller W, Friederich NF (2010) Clinical and radiological outcomes after management of traumatic knee dislocation by open single stage complete reconstruction/repair. BMC Musculoskelet Disord 11:102

28. Kim SJ, Kim SH, Chun YM, Hwang BY, Choi DH, Yoon JY (2012) Clinical comparison of conventional and remnant-preserving transtibial single-bundle posterior cruciate ligament reconstruction combined with posterolateral corner reconstruction. Am J Sports Med 40(3):640-649

29. Yoon KH, Lee JH, Bae DK, Song SJ, Chung KY, Park YW (2011) Comparison of clinical results of anatomic posterolateral corner reconstruction for posterolateral rotatory instability of the knee with or without popliteal tendon reconstruction. Am J Sports Med 39(11): 2421-2428

30. Rios CG, Leger RR, Cote MP, Yang C, Arciero RA (2010) Posterolateral corner reconstruction of the knee: evaluation of a 
technique with clinical outcomes and stress radiography. Am J Sports Med 38(8):1564-1574

31. Freeman RT, Duri ZA, Dowd GS (2002) Combined chronic posterior cruciate and posterolateral corner ligamentous injuries: a comparison of posterior cruciate ligament reconstruction with and without reconstruction of the posterolateral corner. Knee 9(4):309-312
32. Fanelli GC (2006) Surgical treatment of lateral posterolateral instability of the knee using biceps tendon procedures. Sports Med Arthrosc 14(1):37-43

33. Stannard JP, Brown SL, Farris RC, McGwinn G, Volgas DA (2005) The Posterolateral Corner of the Knee Repair Versus Reconstruction. Am J Sports Med 33(6):881-888 